Research

\title{
Radiosensitization by 2-benzoyl-3-phenyl-6,7-dichloroquinoxaline I,4-dioxide under oxia and hypoxia in human colon cancer cells Wafica Itani ${ }^{1}$, Fady Geara ${ }^{2}$, Joelle Haykal ${ }^{1}$, Makhluf Haddadin ${ }^{3}$ and Hala Gali-Muhtasib*1
}

Address: ${ }^{1}$ Department of Biology, American University of Beirut, Beirut, Lebanon, ${ }^{2}$ Department of Radiation Oncology, American University of Beirut, Beirut, Lebanon and ${ }^{3}$ Department of Chemistry, American University of Beirut, Beirut, Lebanon

Email: Wafica Itani - wsi02@aub.edu.lb; Fady Geara -fg00@aub.edu.lb; Joelle Haykal - jmh05@aub.edu.lb; Makhluf Haddadin - haddadin@aub.edu.lb; Hala Gali-Muhtasib* - amro@aub.edu.lb

* Corresponding author

Published: 03 January 2007

Radiation Oncology 2007, 2:1 doi:10.1186/1748-7/7X-2-1
Received: 15 September 2006

Accepted: 03 January 2007

This article is available from: http://www.ro-journal.com/content/2/I/I

(c) 2007 Itani et al; licensee BioMed Central Ltd.

This is an Open Access article distributed under the terms of the Creative Commons Attribution License (http://creativecommons.org/licenses/by/2.0), which permits unrestricted use, distribution, and reproduction in any medium, provided the original work is properly cited.

\begin{abstract}
Background: The sensitizing effects of 2-benzoyl-3-phenyl-6,7-dichloroquinoxaline I,4-dioxide (DCQ) and ionizing radiation (IR) were determined in four colon cancer cells and in FHs74lnt normal intestinal cells.

Methods: Cell cycle modulation, TUNEL assay, clonogenic survival and DNA damage were examined under oxia or hypoxia. Effects on apoptotic molecules and on p-Akt and Cox-2 protein expression were investigated.

Results: The four cell lines responded differently to DCQ+IR; HT-29 cells were most resistant. Combination treatment caused significant increases in preG 1 (apoptosis) in HCT-II6, while $G_{2} / M$ arrest occurred in DLD-I. DCQ potentiated IR effects more so under hypoxia than oxia. Preexposure of DLD-I to hypoxia induced $30 \%$ apoptosis, and $\mathrm{G}_{2} / \mathrm{M}$ arrest in oxia. The survival rate was $50 \%$ lower in DCQ+IR than DCQ alone and this rate further decreased under hypoxia. FHs74Int normal intestinal cells were more resistant to DCQ+IR than cancer cells. Greater ssDNA damage occurred in DLD-I exposed to DCQ+IR under hypoxia than oxia. In oxia, p-Akt protein expression increased upon IR exposure and drug pre-treatment inhibited this increase. In contrast, in hypoxia, exposure to IR reduced $\mathrm{p}$-Akt protein and DCQ restored its expression to the untreated control. Apoptosis induced in hypoxic DLD-I cells was independent of p53-p2I modulation but was associated with an increase in $\mathrm{Bax} / \mathrm{Bcl}-2$ ratio and the inhibition of the Cox-2 protein.
\end{abstract}

Conclusion: DCQ is a hypoxic cell radiosensitizer in DLD-I human colon cancer cells.

\section{Background}

Oxygen is known to help in stabilizing the radiationinduced DNA damage [1]. The lack of oxygen in solid malignant tumors results in their resistance to radiation therapy $[1,2]$. Attempts to overcome this resistance include the use of "oxygen-mimetic" radiosensitizers [3]; compounds which offer an attractive alternative for increasing the therapeutic window [4]. 
Quinoxaline 1,4-dioxides (QdNOs) share the di-N-oxide moiety with the clinically used drug Tirapazamine. These hypoxia-selective compounds are known to be redox-activated DNA-cleaving agents [5]. DNA cleavage by QdNOs requires enzymatic one-electron reduction of the compound to an activated, oxygen-sensitive intermediate [6]. This one-electron reduction is more likely to occur in the reducing conditions of hypoxic cells, targeting the toxicity of these compounds to hypoxic cells. Recent studies have shown that the nature of the substituent on the benzoring of the QDNO influences its potency [7]. Mild electron withdrawing groups in the 6(7) position increase the potency of these compounds under hypoxic conditions [7].

We have shown that the compound, 2-benzoyl-3-phenyl6,7-dichloroquinoxaline 1,4-dioxide (DCQ), is a hypoxic cytotoxin [8]. Treatment of human colon cancer T-84 cells with DCQ reduced the expression levels of HIF- $1 \alpha$ mRNA and protein [8]. The decrease in HIF- $1 \alpha$ mRNA and protein expression by DCQ was later documented in EMT6 mouse mammary adenocarcinoma and Lewis lung carcinoma cells [9]. DCQ was also shown to reduce the expression levels of vascular endothelial growth factor (VEGF) and to inhibit hypoxia-induced angiogenesis [9]. Subsequent experiments performed by our group established that DCQ is an effective radiosensitizer both in vitro and in vivo [9]. When DCQ was combined with radiation, doses of 2.5-5 $\mu \mathrm{M}$ resulted in a dramatic decrease in clonogenic survival of EMT6 cells. The mechanism of radiosenitization by DCQ in EMT6 cells was found to involve the induction of $\mathrm{G}_{2} / \mathrm{M}$ arrest and apoptosis (unpublished results). Radiosensitization effects were also seen in vivo when LLC tumors were injected into C57BL/J6 mice and the effects of DCQ+IR on tumor volume were observed over 20 days [9].

This study aims, for the first time, to determine DCQ radiosensitizing activities in several human colorectal cancer cell lines and to investigate its cell cycle modulatory effects under both oxic and hypoxic conditions. Drug sensitization was examined in the FHs74Int normal human intestinal cell line to determine the sensitivity of normal cells to DCQ. In addition, the DNA damaging potential of DCQ and its effects on the protein expression levels of the oncogene Akt and on key molecules of apoptosis was investigated.

\section{Methods}

\section{Cell culture}

FHs74Int normal human intestinal cells were cultured in Hybri-Care medium supplemented with $30 \mathrm{ng} / \mathrm{ml}$ epidermal growth factor. Human colon cancer cell lines (DLD1, HT-29, HCT-116, and SW-480) were grown in RPMI 1640 containing L-Glutamine and $25 \mathrm{~mm}$ HEPES. All media were supplemented with $10 \%$ heat-inactivated FBS and $1 \%$ Penicillin-Streptomycin $(50 \mu \mathrm{g} / \mathrm{ml})$. Cells were cultured in a humidified incubator $\left(95 \%\right.$ air $\left.5 \% \mathrm{CO}_{2}\right)$ at $37^{\circ} \mathrm{C}$ (Forma Scientific Inc. Ohio, USA).

\section{Drug preparation}

DCQ was synthesized from 5,6-dichlorobenzofurazan oxide and dibenzoylmethane according to the Beirut Reaction [10]. A fresh stock of $10 \mathrm{mg}$ of DCQ was dissolved in $1 \mathrm{ml}$ of filtered DMSO. Before treatment, DCQ was diluted 1 in 10 using media containing 10\% FBS and $1 \%$ Penicillin-Streptomycin $(50 \mu \mathrm{g} / \mathrm{ml})$.

\section{Radiation experiments}

Cells cultured in $25 \mathrm{~cm}^{2}$ T-flasks were treated either with DCQ $(0-10 \mu \mathrm{M})$, irradiation (0-6 Gy) or combinations. Irradiation was administered by a JL Shepherd, 143-68 Cesium-137 Laboratory Irradiator with an output activity of $1683 \mathrm{Ci}$. Immediately after irradiation, cells were replenished with fresh media containing no drugs and left in the incubator for 24 hours for studies on cell cycle regulation and DNA damage (COMET) as described below.

\section{Hypoxia treatment}

DLD-1 or FHs 74Int cells cultured in $25 \mathrm{~cm}^{2}$ flasks were treated at $50 \%$ confluency with DCQ for 4 hours, after which they were placed in a tightly sealed chamber $\left(37^{\circ} \mathrm{C}\right.$, $1 \% \mathrm{O}_{2}$ ) for 1 hour. The desired oxygen level was optimized by injecting $\mathrm{N}_{2}$ gas into the chamber, and the levels were measured every 15 minutes using an Ohmeda Oxymeter (Datex-Ohmeda, Louisville, CO). Immediately after hypoxia the flasks were sealed and the cells were irradiated. Later, cells were replenished with fresh media containing no drugs and incubated for another 24 hours.

\section{Clonogenic survival}

Oxic or hypoxic DLD-1 cells cultured in $25 \mathrm{~cm}^{2} \mathrm{~T}$-flasks were treated with DCQ $(0-100 \mu \mathrm{M}, 1$ hour $)$, after which they were irradiated (2 Gy). FHs74Int cells were treated under oxic conditions with DCQ $(0-10 \mu \mathrm{M})$ for 1 hour prior to irradiation (2 Gy). Immediately after irradiation, both cell lines were re-plated at known dilutions with fresh media for 10 days. After 10 days of incubation, colonies were stained with crystal violet and counted. The number of colonies containing more than 50 cells was counted and the percentage of survival rates at each dose was calculated according to the formula: (colony no. in treatment/colony no. in control) $\times 100$.

\section{Cell cycle analysis using flow cytometry}

Following treatment, cells were harvested, fixed in ice cold $70 \%$ ethanol and stored at $-20^{\circ} \mathrm{C}$. On the day of DNA staining, cells were incubated for 75 minutes in $200 \mu \mathrm{g} / \mathrm{ml}$ RNase A at $37^{\circ} \mathrm{C}$, and stained with $50 \mu \mathrm{g} / \mathrm{ml}$ propidium iodide. Cell cycle analysis was performed using a FACScan 
flow cytometry (Becton Dickinson, Research Triangle, $\mathrm{NC}$ ) and the percentage of cells in pre $\mathrm{G}_{1}, \mathrm{G}_{1}, \mathrm{~S}$, and $\mathrm{G}_{2} / \mathrm{M}$ phases was determined using the Cell Quest program.

\section{Apoptosis TUNEL assay}

Fragmented DNA was detected by Terminal deoxy-transferase (TdT)-mediated dUTP nick-end labeling (TUNEL assay) (Roche Diagnostics, Mannheim, Germany) to assess the induction of apoptosis. Following treatment, cells were harvested and the pellet was suspended in 100 $\mu \mathrm{l}$ freshly prepared PBS in $4 \%$ formaldehyde, incubated at room temperature for 30 minutes, and centrifuged at 300 $\mathrm{g} / 2000 \mathrm{rpm}$ for 10 minutes. The pellet was washed once with $200 \mu \mathrm{l}$ PBS. Followed by suspension in $100 \mu \mathrm{l}$ of a solution containing $1 \times$ PBS, $0.1 \%$ sodium citrate, and $0.1 \%$ Triton X-100 for 2 minutes on ice. Cells were then washed twice with $1 \times$ PBS. The pellet was resuspended in $50 \mu \mathrm{l}$ tunnel reaction mixture ( $45 \mu \mathrm{l}$ labeling solution and $5 \mu$ l enzyme solution), incubated for $1 \mathrm{~h}$ at $37^{\circ} \mathrm{C}$ in a humidified atmosphere in the dark, then washed twice with $1 \times$ PBS and suspended in $1 \times$ PBS for reading by flow cytometry. Cells suspended in $50 \mu$ labeling solution served as the negative control. The samples were examined by FACScan flow cytometer to determine the percentage of apoptotic cells in treated samples as compared to the control samples.

\section{Single Cell Gel Electrophoresis (SCGE)/comet assay}

DNA damage, including single strand breaks (SSB) and alkali labile sites (ALS), was measured using the alkaline SCGE assay in DLD-1 cells treated with DCQ $(5 \mu \mathrm{M}, 1$ hour) IR (2 Gy) or combinations under oxia or hypoxia. Immediately after IR, cells were scraped and collected in RPMI medium. Comet assay was performed as described previously [11]. For electrophoresis, an electric current of 25 volts and $300 \mathrm{~mA}$ was applied for 30 minutes, after which the slides were placed in a neutralizing buffer for 5 minutes. This neutralizing procedure was repeated two more times. Finally, $50 \mu \mathrm{l}$ of YOYO stain $(0.25 \mu \mathrm{M}$ YOYO, $2.5 \%$ DMSO and $0.5 \%$ sucrose) (Molecular Probes Eugene, Oregon, USA) was added to each slide and analyzed immediately using a fluorescence microscope (AXIOVERT 200, ZEISS Flourescence and optical microscope with ZEISS AXIOCAM HRC and KS 300 V3 image analysis software). Images of 100 randomly selected nonoverlapping cells (magnification 100x) were analyzed for each sample with the help of Tri-Tek CometScore ${ }^{\mathrm{TM}}$ software, a fully automatic image analysis system. The following parameters were used to assess DNA damage: total fluorescence of the comet, fluorescence of the tail, percentage of DNA in the tail region and tail moment (\%DNA in tail multiplied by tail length). The comet data values were expressed as mean \pm S.D. Statistical comparisons were made by $t$-test and the $P$-values $<0.05$ or $P<$ 0.01 were considered significant.

\section{Protein expression by Western Blotting}

DLD-1 cells cultured in $75 \mathrm{~cm}^{2}$ T-flasks were treated with DCQ ( $5 \mu \mathrm{M}, 1$ hour), IR (2 Gy) or combinations under oxic or hypoxic conditions. Cellular proteins were extracted by SDS-lysis buffer (50 mM Tris-HCL, pH 7.5, $150 \mathrm{mM} \mathrm{NaCl}, 1 \%$ Nonidet P40, 0.5\% Sodium deoxycholate, $4 \%$ protease inhibitors and 1\% phosphatase inhibitors). Protein extracts were centrifuged for 10 minutes at $14,000 \mathrm{rpm}$. Proteins were quantified using the DC BioRad Protein Assay kit with BSA as a standard. Whole cell lysates (40-60 $\mu \mathrm{g}$ ) were loaded on 12\% SDS-polyacrylamide gels and then transferred onto PVDF membranes (Amersham Pharmacia Biotech, Amersham, England). The membranes were incubated with the primary antibodies: p21 (F-5), p53 (FL-393), p-p53, Bcl-2 (N-19), Cox-2 (all from Santa Cruz, CA), Bax (Biosource, California, USA), pS473 Akt (44-622G) (Chemicon International, California, USA). The GAPDH antibody (Biogenesis, Poole, UK) was used as a loading control. The membrane was then washed 3 times for 10 minutes each in wash buffer (TBS containing 0.05\%-0.1\% Tween 20) and probed with the appropriate secondary antibody (IgG-HRP, antirabbit IgG-HRP, or antigoat IgG-HRP from Santa Cruz) for 1 hour at room temperature. After wash, the membrane was exposed to X-ray film (Hyperfilm ECL, Lebanon) using a chemiluminescent substrate (Amersham Pharmacia Biotech, Amersham, England). The bands were quantified using LabWorks 4.0 software.

\section{Results \\ Cell cycle modulation in four human colon cancer cell lines under oxia}

To study cell cycle modulation by DCQ+IR, cells were incubated with DCQ ( 5 or $10 \mu \mathrm{M}$ ) for either 1 hour (DLD1 and HCT116) or 4 hours (SW-480 and HT-29), and then irradiated (2 Gy). The times, 1 or 4 hours, were chosen based on differences in the sensitivity of the four cell lines to the drug. While SW-480 and HT-29 survived after 4 hour exposure to DCQ, DLD-1 and HCT-116 died when drug treatment was extended for more than 1 hour (data not shown). Twenty four hours after treatment, cells were harvested for flow cytometry analysis and the percentage of cells in pre $G_{1}$ and $G_{2} / M$ phases were plotted as these phases were the most modulated. The response of the four cell lines to DCQ+IR was different; HT-29 cells were the most resistant followed by SW-480 (Figure $1 \mathrm{~A}$ and $1 \mathrm{~B}$ ). HCT116 and DLD-1 were sensitive to DCQ+IR, but responded differently. Treatment with $10 \mu \mathrm{M} D C Q+I R$ caused 11 fold increases in the preG $\mathrm{g}_{1}$ portion in HCT-116 (Figure 1C), however, in DLD-1 cells 2-fold increases in the percentage of $\mathrm{G}_{2} / \mathrm{M}$ cells was observed (Figure $1 \mathrm{D}$ ). 

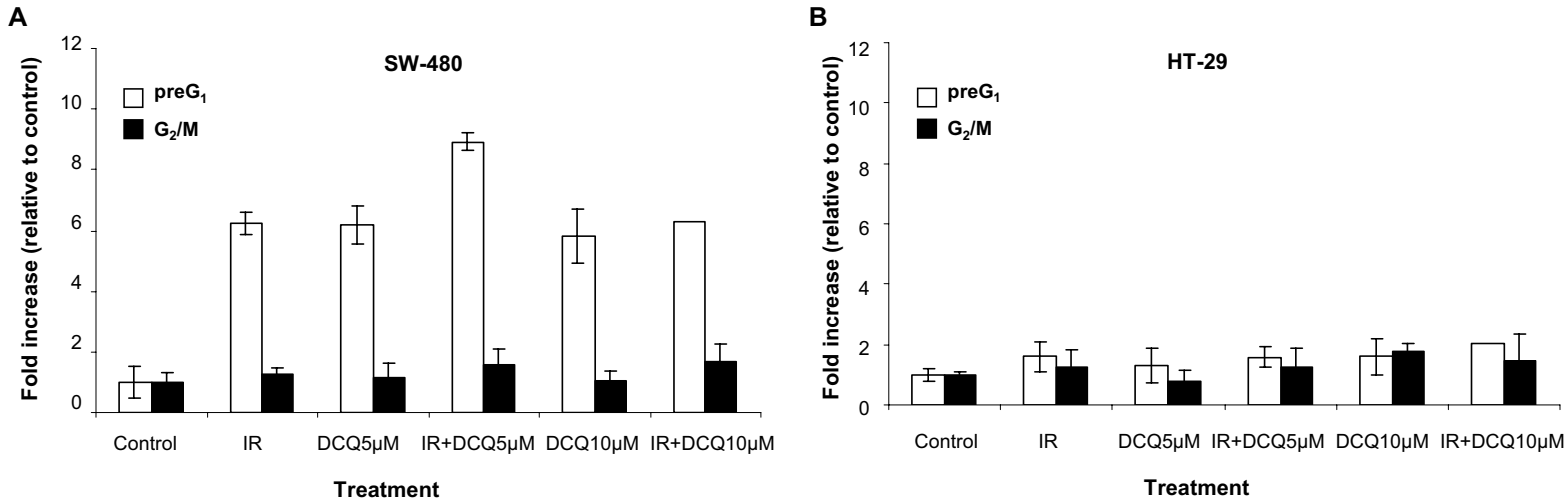

C

D
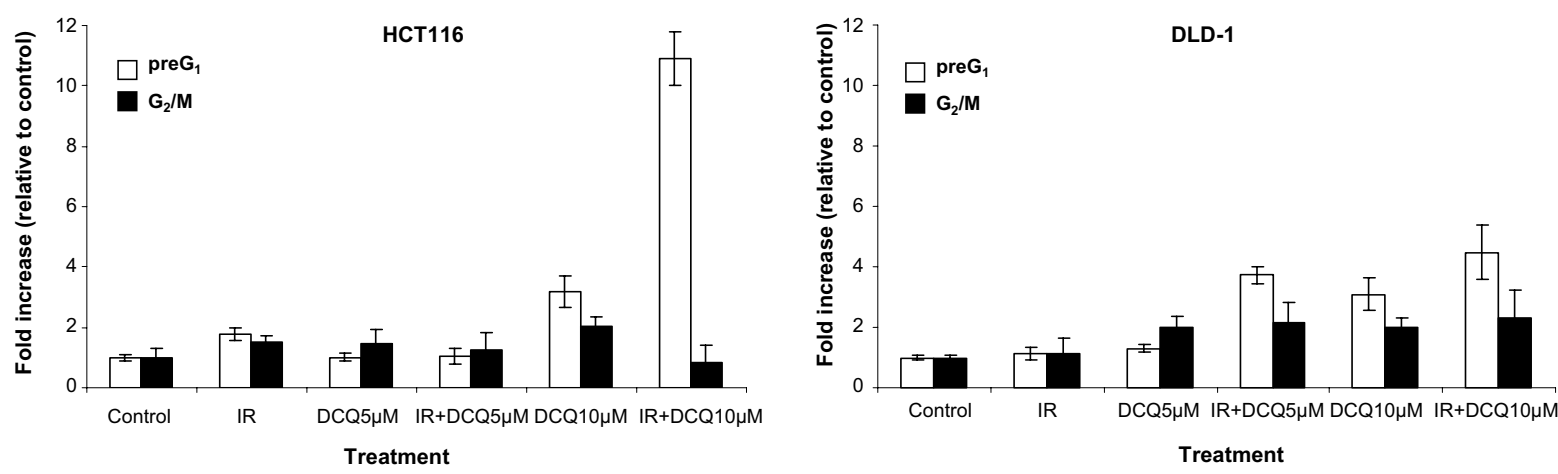

Figure I

Effect of DCQ, IR and their combinations on cell cycle regulation in four different human colon cancer cell lines (SW-480, HT29 , HCTII 6 and DLD-I). Cells were treated with DCQ $(0,5,10 \mu \mathrm{M})$, IR (2 Gy) or combinations. Immediately after radiation or drug treatment, cells were replenished with fresh medium containing no drug and incubated for another 24 hours. Control cells were treated with DMSO (0.1\%). Cell cycle changes were assessed using Propidium lodide stain with flow cytometry as described in "Materials and Methods". The percentage of cells in preG, and $G_{2} / M$ phases were plotted as a function of $D C Q$ dose. Results are representative of at least two independent experiments each performed in duplicates.

\section{Cell cycle modulation in HCTI 16 and SW-480 cells under hypoxia}

Since DCQ is a hypoxic cytotoxin [9], we then investigated whether it could potentiate IR effects more so under hypoxia than oxia. The hypoxia toxicity of DCQ was first studied in the two cell lines, HCT116 and SW-480. Cells were incubated in DCQ ( $5 \mu \mathrm{M}, 1$ or 4 hours) under oxic or hypoxic conditions, after which they were irradiated, then replenished with media containing no DCQ, and harvested 24 hours later for cell cycle analysis (Figures 2 and 3). In both cell lines, hypoxia treatment alone caused $\mathrm{G}_{2} / \mathrm{M}$ arrest (1.5-2.0 fold increase). Exposure of HCT-116 cells to oxic or hypoxic conditions prior to IR resulted in no difference in their sensitivity to the drug (\% of cells in preG $_{1}$ phase was $36 \%$ in oxia and $23 \%$ in hypoxia) (Figure 2). However, SW-480 showed a significant increase in pre $_{1}$ cells when combination treatment was done under hypoxia (Figure 3). Considering that HCT116 and SW480 were sensitive to hypoxia, no further studies were done with these cell lines.

\section{Cell cycle modulation and clonogenic survival in DLD-I cells under hypoxia}

To investigate the hypoxic cytotoxicity of DCQ in DLD-1, we compared its efficacy in cells incubated in oxia or hypoxia prior to irradiation. DLD-1 cells were treated with 


\section{HCT116}
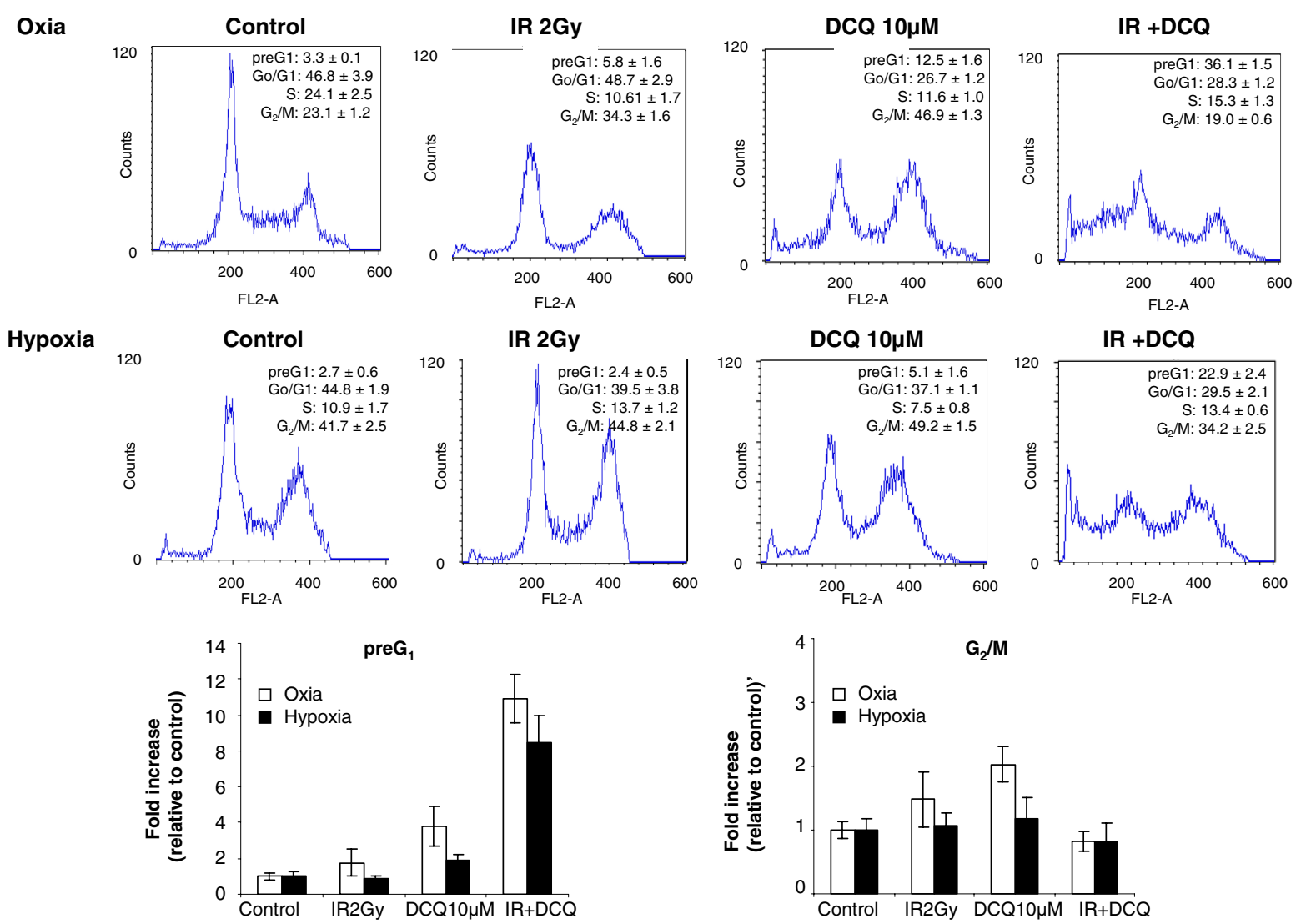

\section{Figure 2}

Effect of DCQ, IR and their combinations on cell cycle regulation in HCTI I 6 cells exposed to oxic or hypoxic conditions. Cells were treated with $5 \mu \mathrm{M}$ DCQ or DMSO $(0.1 \%)$ and exposed to hypoxia or incubated in oxia for I hour, then irradiated $(2$ Gy). Immediately after radiation or drug treatment, cells were replenished with fresh medium containing no drug and incubated for another 24 hours. Cell cycle changes were assessed using Propidium iodide stain with flow cytometry as described in "Materials and Methods". Bar graphs are a summary of at least three independent experiments each performed in duplicates.

DCQ $(5 \mu \mathrm{M})+$ IR and harvested after 24 hours for cell cycle analysis (Figure 4). Treatment under oxia resulted in the accumulation of $63 \%$ of the cells in $\mathrm{G}_{2} / \mathrm{M}$ phase and $4 \%$ in preG $_{1}$. More pronounced effects were observed in hypoxia, as $33 \%$ of apoptotic cells accumulated in preG (Figure 4). Therefore treatment of DLD-1 cells with DCQ+IR caused $G_{2} / M$ arrest in oxia and preG1 arrest in hypoxia.

Using TUNEL assay, the level of apoptosis in cells treated with DCQ+IR under oxic and hypoxic conditions was found to be $3.9 \%$ and $30 \%$ respectively (Figure 5) confirming that the increases in preG1 observed by flow cytometry are due to apoptosis.

To confirm the hypoxic effects of DCQ, DLD-1 cells were treated with DCQ $(1-100 \mu \mathrm{M})$ in oxia or hypoxia, irradi- ated (2 Gy) and then re-plated at known dilutions. Ten days after re-plating, the surviving colonies were counted. The survival curves for DCQ+IR and DCQ alone show a more pronounced decrease in cell survival under hypoxia than oxia (Figure 6). Exposing DLD-1 cells to IR alone did not reduce the absolute survival rate of cells under hypoxia as compared to oxia (Figure 6C). When DLD-1 cells were exposed to DCQ alone $(10 \mu \mathrm{M})$, the surviving fraction determined with respect to the untreated cells was $0.49(\mathrm{SD} \pm 0.04)$ in oxia and $0.20(\mathrm{SD} \pm 0.02)$ in hypoxia (Figure 6A). However, when DCQ $(10 \mu \mathrm{M})$ was combined with IR, the surviving fraction determined with respect to the irradiated cells dropped to $0.29(S D \pm 0.03)$ in oxia and $0.04(\mathrm{SD} \pm 0.01)$ in hypoxia (Figure $6 \mathrm{~B})$.

The hypoxia cytotoxicity ratio (HCR), i.e. the concentration of drug required under oxia relative to hypoxia to 

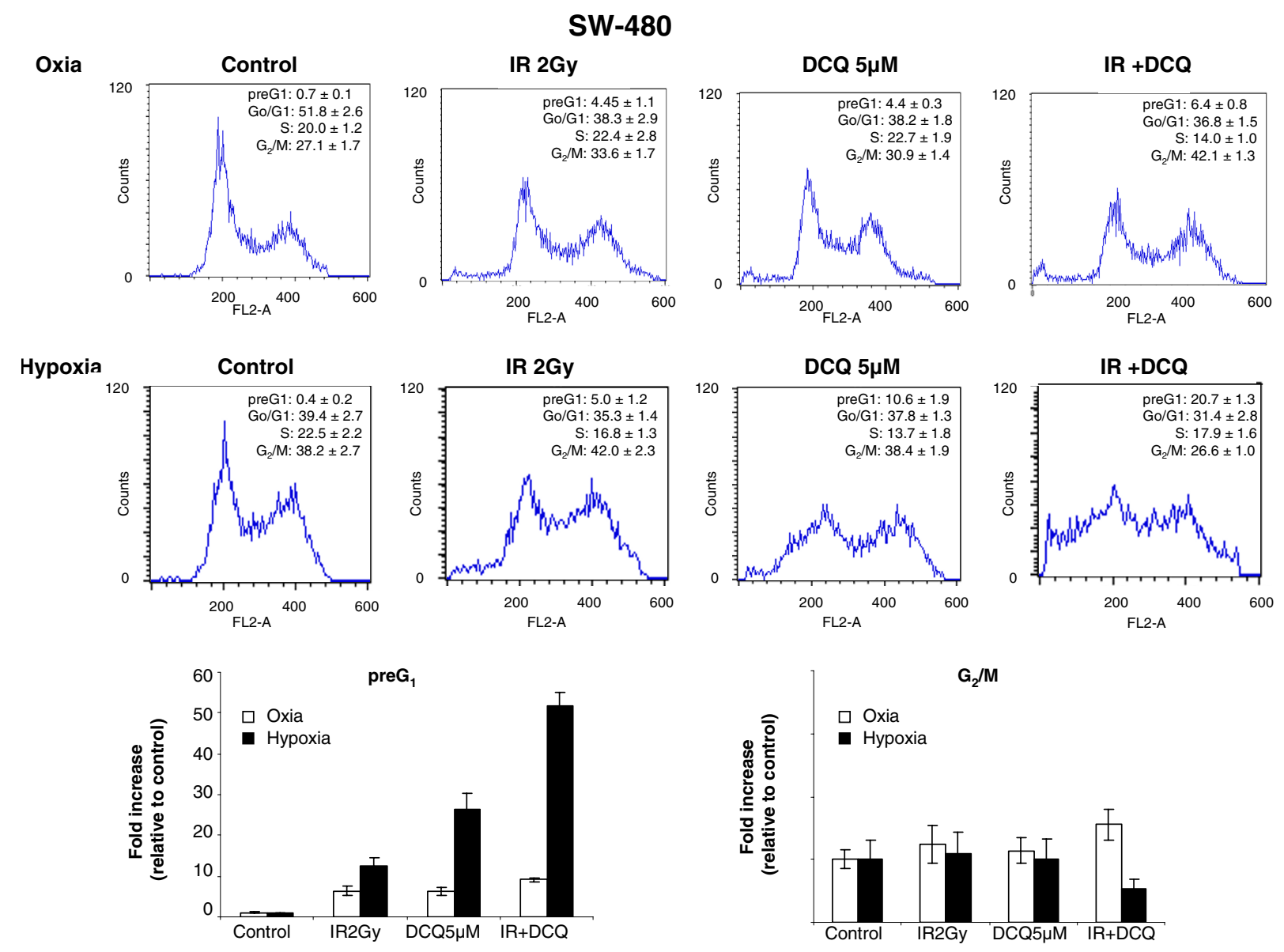

\section{Figure 3}

Effect of DCQ, IR and their combinations on cell cycle regulation in SW-480 cells exposed to oxic or hypoxic conditions. Cells were treated with $5 \mu \mathrm{M} \mathrm{DCQ}$ or DMSO $(0.1 \%)$ and exposed to hypoxia or incubated in oxia for 4 hours, then irradiated $(2$ Gy). Immediately after radiation or drug treatment, cells were replenished with fresh medium containing no drug and incubated for another 24 hours. Cell cycle changes were assessed using Propidium iodide stain with flow cytometry as described in "Materials and Methods". Bar graphs are a summary of at least three independent experiments each performed in duplicates.

produce $90 \%$ cell death, was 4 fold higher when DCQ was combined with IR $(\mathrm{HCR}=12)$ as compared to DCQ alone $(\mathrm{HCR}=3)$. This provided additional evidence that the drug is a potent radio-sensitizer in hypoxic cells.

\section{DCQ radiosensitization in the FHs74Int normal intestinal cell line}

After establishing effects of DCQ and IR in cancer cells, we compared DCQ efficacy in normal cells. For this purpose, FHs74Int normal human intestinal cells were pre-treated with DCQ (1.25-10 $\mu \mathrm{M}, 1$ hour), irradiated, and then replated at known dilutions and the surviving colonies were determined 10 days later. At $5 \mu \mathrm{M} \mathrm{DCQ}$, the survival rate was 0.68 ( $\mathrm{SD} \pm 0.02)$, and this rate was reduced to 0.46 $(\mathrm{SD} \pm 0.01)$ when DCQ $(5 \mu \mathrm{M})$ was combined with IR (Figure 6D). A comparison of the extent of decrease in cell survival in DCQ+IR in normal FHs74Int v.s. DLD-1 cancer cells confirms the greater radio-sensitizing effects of this drug in cancer cells.

\section{DNA damage by DCQ in irradiated DLD- I cells under oxia and hypoxia}

To determine if DCQ is a DNA-targeting agent, the extent of DNA damage was measured by the alkaline COMET assay in oxic or hypoxic DLD-1 cells exposed to DCQ (5 $\mu \mathrm{M}, 1$ hour), IR or combinations. The COMET assay measures single strand DNA breaks by the increase in the electrophoretic mobility of denatured genomic DNA in an agarose gel. Figure 7A shows an example of different grades of DNA fragmentation. In the first image, the DNA of a largely non-fragmented cell is depicted. The next 2 images represent cells with increasingly fragmented DNA; thus giving the comet its tail. The last image shows a cell with highly fragmented DNA. Treatment with DCQ+IR 


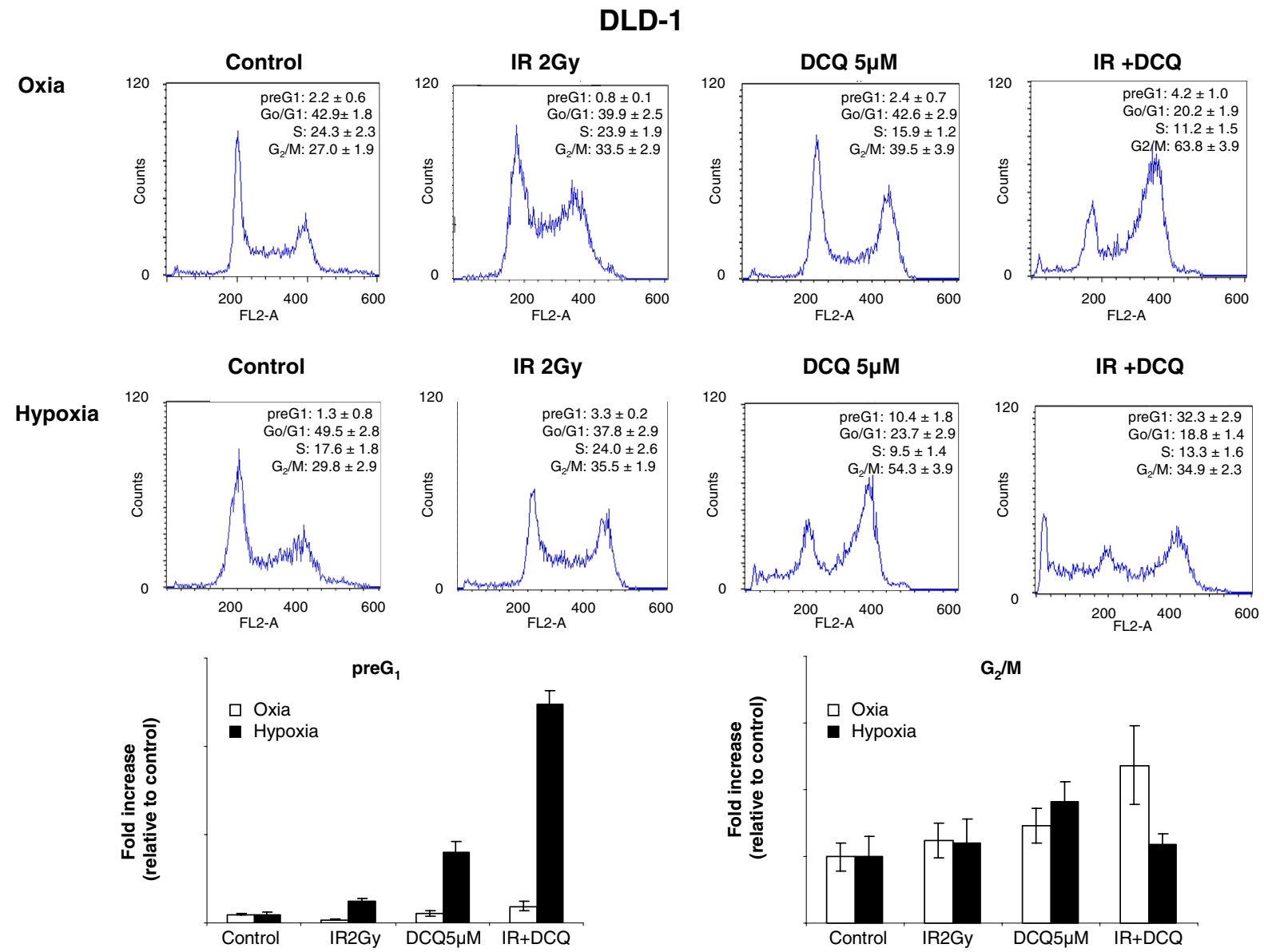

\section{Figure 4}

Combination effects of DCQ and IR in DLD-I cells under oxic and hypoxic conditions. Cells were treated with $5 \mu M$ DCQ or DMSO $(0.1 \%)$ and exposed to hypoxia or incubated in oxia for I hour, then irradiated (2 Gy). Immediately after radiation or drug treatment, cells were replenished with fresh medium containing no drug and incubated for another 24 hours. Cell cycle changes were assessed using Propidium iodide stain with flow cytometry as described in "Materials and Methods". Bar graphs are a summary of at least three independent experiments each performed in duplicates.

resulted in a statistically significant increase $(p<0.01)$ in DNA damage in hypoxia compared to oxia. The mean percentage of DNA damage was $95(S D \pm 5.65)$ in cells exposed to DCQ+ IR under hypoxia as compared to only $60.5(\mathrm{SD} \pm 2.12)$ under oxia (Figure $7 \mathrm{~B})$.

Digital images were further analyzed using Comet Score software that allows quantitative measurements of various comet assay end-points, in particular, the mean average of comet length, tail length, and percentage of DNA in the tail (Figure 7C). In addition, tail moment was calculated as the product of the percentage of DNA in the comet tail multiplied by the total comet length. Such endpoints are the most accepted parameters for assessing DNA damage. It is important to note that 1 hour exposure of the cells to hypoxia did not induce a major change in any of the measured comet assay end-points.
Several end-point measures indicated that DCQ is a more potent DNA damaging agent in irradiated hypoxic cells: $1)$ significant $(p<0.05)$ increase in mean tail moment in hypoxia compared to oxia (24.69 in oxia v.s. 72.3 in hypoxia); 2) greater relative amount of damage, quantified by measuring the distance that DNA moves in the gel or the length of the comet tail; 3 ) greater amount of DNA present in the tail in hypoxic cells ( 11 fold increase in tail DNA in hypoxia v.s. 7 -fold increase in oxia) (Figure 7C).

\section{DCQ effects on radiation-induced $p 53, p-p 53$ and $p 2$ I expression}

To investigate the effects of DCQ on key apoptotic molecules, DLD-1 cells were treated with DCQ, IR or combinations under oxic or hypoxic conditions and the expression levels of p53, p-p53 and p21 proteins were determined (Figure 8 ). The phosphorylation of p53 normally stabi- 


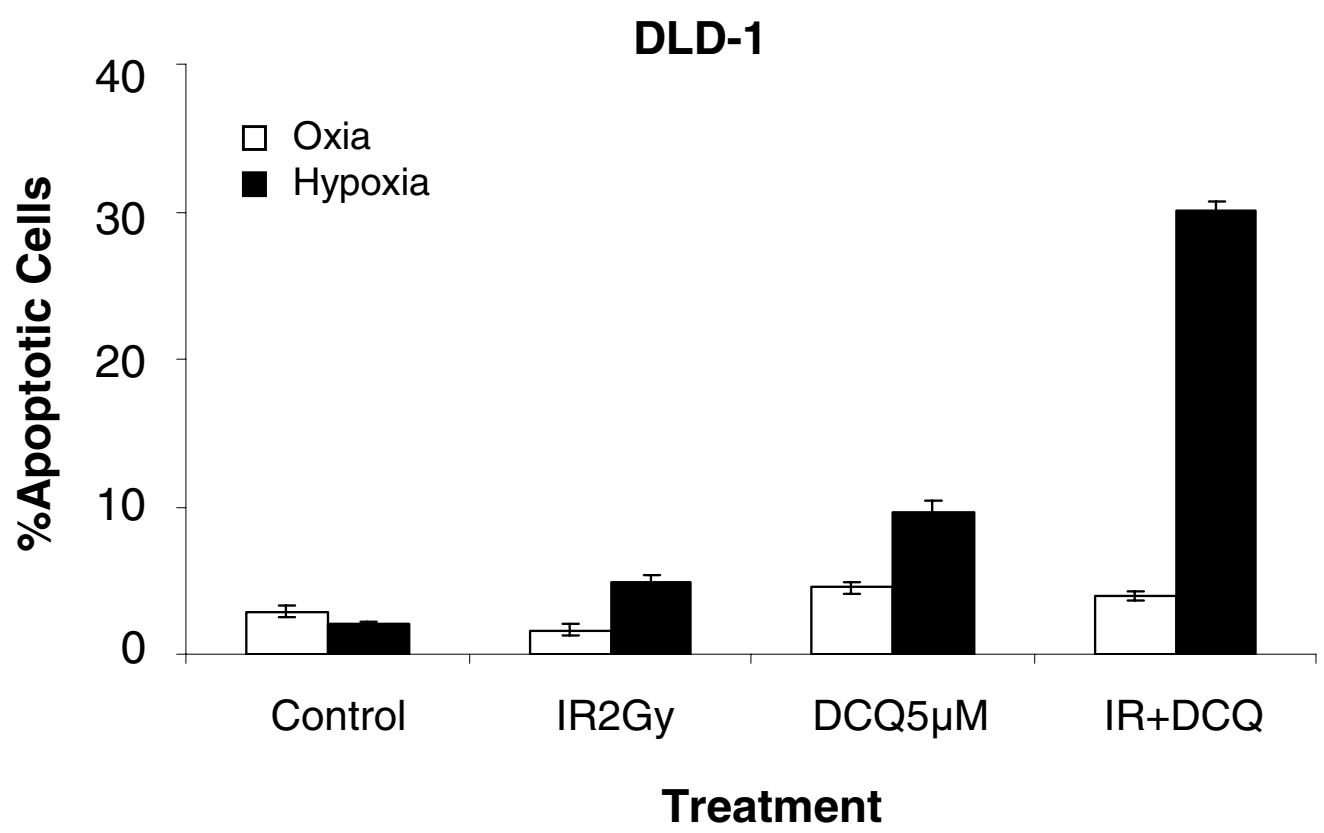

\section{Figure 5}

TUNEL assay showing that the combination of DCQ and IR induces apoptosis in DLD-I cells under oxic and hypoxic conditions. Cells were treated with $5 \mu \mathrm{MDCQ}$ or DMSO $(0.1 \%)$ and exposed to hypoxia or incubated in oxia for I hour, then irradiated (2 Gy). Immediately after radiation or drug treatment, cells were replenished with fresh media containing no drugs and left in the incubator for 24 hours. The extent of DNA fragmentation was determined by TUNEL assay and measured by flow cytometry. The percentage of apoptotic cells was determined using CellQuest. Results are representative of at least two independent experiments.

lizes the protein $[12,13]$ which in turn activates and stabilizes p21 leading to cell cycle arrest [14,15]. In hypoxia, the IR-induced p53 protein expression levels were reduced by 0.3 fold in cells exposed to DCQ prior to IR (Figure 8A). A much greater increase in the expression levels of $\mathrm{p}$ p53 protein was evident in cells exposed to DCQ+IR under oxia ( 8 fold) than hypoxia (1.3 fold) (Figure $8 \mathrm{~A}$ ). This increase was associated with an increase in p21 protein expression levels under oxia (3.7 fold) and hypoxia (1.5 fold) (Figure 8A). This finding aligns with the fact that the induction of p21 under hypoxia may be independent of p53 status.

\section{DCQ effects on radiation-induced $\mathrm{Bax} / \mathrm{BCl}-2$ expression}

We then investigated whether DCQ radiosensitization is associated with changes in the levels of the anti-apoptotic Bcl-2 and pro-apoptotic Bax proteins. Up regulation of Bax and down regulation of $\mathrm{Bcl}-2$ favor the pro-apoptotic over the anti-apoptotic response in the cell leading to the release of cytochrome $c$ and promoting cell death. Treatment with DCQ+IR in oxic cells did not induce changes in the $\mathrm{Bax} / \mathrm{Bcl}-2$ ratio (Figure $8 \mathrm{~B}$ ). However, $\mathrm{DCQ}+\mathrm{IR}$ in hypoxic cells increased Bax/Bcl-2 expression by 2.3 fold.

\section{DCQ effects on radiation-induced $p$-Akt expression}

Since the Akt survival oncogene is known to be involved in the transition to $G_{2} / M[16]$, its inhibition may lead to cell cycle arrest at $\mathrm{G}_{2} / \mathrm{M}$ phase. In oxic cells, p-Akt protein expression levels increased upon exposure to IR; pretreatment with DCQ inhibited this increase in $\mathrm{p}$-Akt protein (Figure 8C). In contrast, in hypoxic cells, exposure to IR reduced p-Akt protein expression levels and DCQ restored those levels to the untreated control (Figure 8C). It appears that the inhibition of p-Akt by DCQ under oxia results in enhanced susceptibility of DLD-1 cells to IR, thus leading to cell cycle arrest at $\mathrm{G}_{2} / \mathrm{M}$.

\section{DCQ effects on radiation-induced Cox-2 expression}

Cox-2 is an anti-apoptotic protein the expression of which is reduced at high $\mathrm{Bax} / \mathrm{Bcl}-2$ protein expression levels [17]. Therefore, we examined whether DCQ radiosensitization is associated with changes in the Cox-2 protein (Figure 8). Recent studies show that Cox-2 inhibition can restore p53 levels in response to hypoxia and thereby render the cells more sensitive to therapeutic agents [18]. DLD-1 cells exposed to hypoxia had 1.7 fold higher levels of Cox-2 protein than those exposed to oxia (Figure 8C). Pre-treatment with DCQ was found to inhibit the IR-induced lev- 

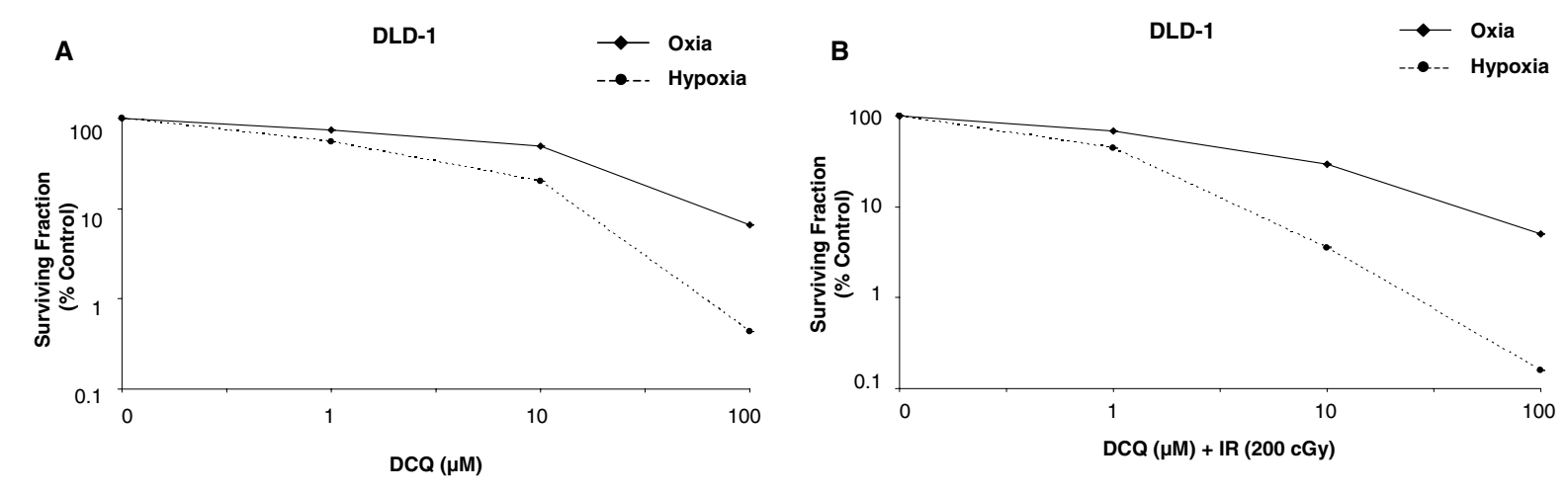

C

\begin{tabular}{|l|c|c|c|c|c|c|c|}
\cline { 3 - 8 } \multicolumn{1}{c|}{} & \multicolumn{3}{c|}{ DCQ } & \multicolumn{3}{c|}{ DCQ + IR 2Gy } \\
\cline { 2 - 8 } \multicolumn{1}{c|}{} & IR 2Gy & $1 \mu \mathrm{M}$ & $10 \mu \mathrm{M}$ & $100 \mu \mathrm{M}$ & $1 \mu \mathrm{M}$ & $10 \mu \mathrm{M}$ & $100 \mu \mathrm{M}$ \\
\hline Oxia & 0.61 & 0.43 & 0.29 & 0.04 & 0.42 & 0.18 & 0.031 \\
\hline Hypoxia & 0.52 & 0.32 & 0.12 & 0.0026 & 0.27 & 0.022 & 0.001 \\
\hline
\end{tabular}

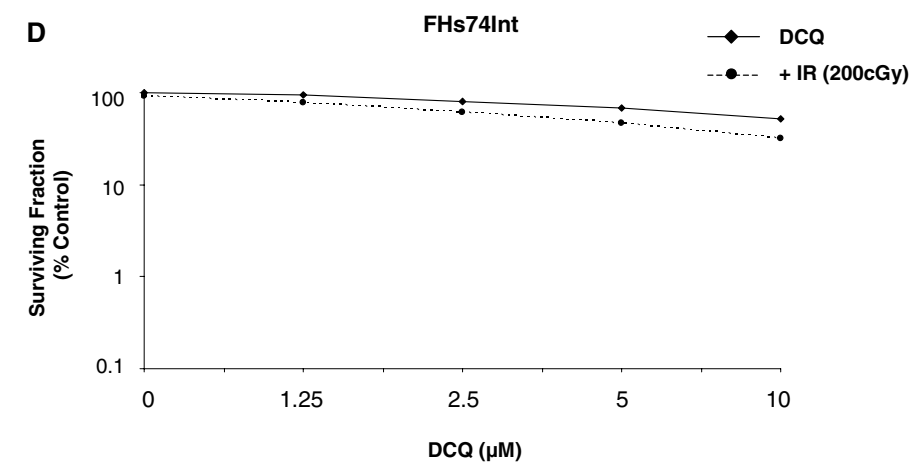

\section{Figure 6}

Survival curves of DLD-I cancer cells and FHs74Int normal cells exposed to DCQ alone or DCQ and irradiation. A. DLD-I cells were exposed to I hour oxia or hypoxia in the presence of DCQ and the surviving fraction was determined as a percentage with respect to the untreated cells. B. DLD-I cells were exposed to I hour oxia or hypoxia in the presence of DCQ and then irradiated $(2 \mathrm{~Gy})$ and the surviving fraction was determined as a percentage with respect to the irradiated cells. C. Absolute survival rates of DLD-I cells exposed to DCQ, IR or their combinations under oxic and hypoxic conditions. D. FHs74Int cells were exposed to I hour oxia in the presence of DCQ and then irradiated. After irradiation, cells were re-plated and the colonies were stained with crystal violet and counted 10 days later. Each data point was calculated as percent of untreated cells of two independent experiments each performed in duplicates.

els of Cox-2 protein by 0.2 fold in oxic cells and by 9.8 fold in hypoxic cells. It is interesting to note that the significant inhibition of Cox-2 protein by DCQ in hypoxic and irradiated cells is associated with increased p-p53 protein levels and Bax/Bcl-2 ratio (Figure $8 \mathrm{C}$ ). Such protein modulation may be responsible for the greater DCQ radiosensitization in hypoxic cells.

\section{Discussion}

The use of non-toxic drugs that are activated in hypoxic regions of tumors are known to enhance the killing effects of radiation therapy and to be the most effective treatment modality so far [19]. Here, we demonstrate that DCQ is a DNA-damaging radiosensitizer with greater efficacy towards hypoxic tumor cells. This is the first report of DCQ sensitization when combined with IR against human colon cancer cells.

All four human colon cancer cell lines were sensitive to DCQ+IR, but to a different extent. Although HT-29 cell line was resistant, the three other cell lines (HCT116, SW480, DLD-1) showed relative sensitivity towards the combination of DCQ and radiation. The efficacy of the drug was enhanced when the cells were exposed to hypoxia prior to irradiation. The combination of drug and radiation treatment under hypoxia resulted in apoptosis, while such treatment induced $G_{2} / M$ arrest in oxic cells. This indicates that DCQ enhances IR effects to a different 
A

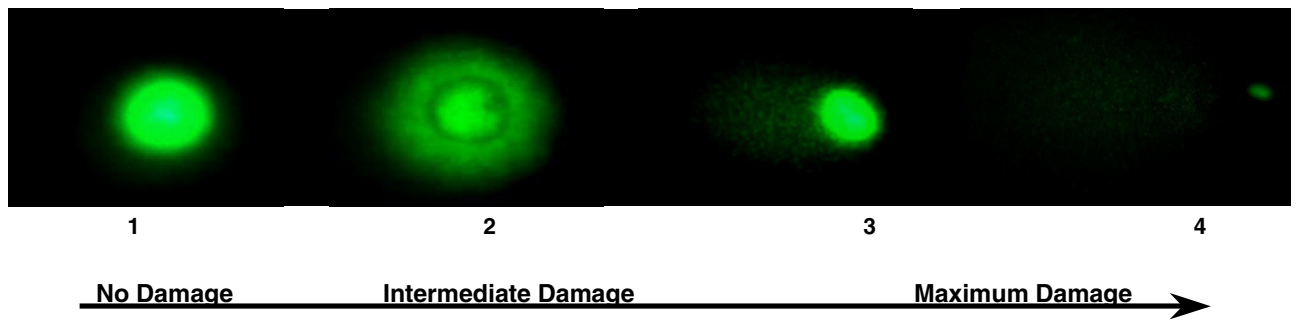

B

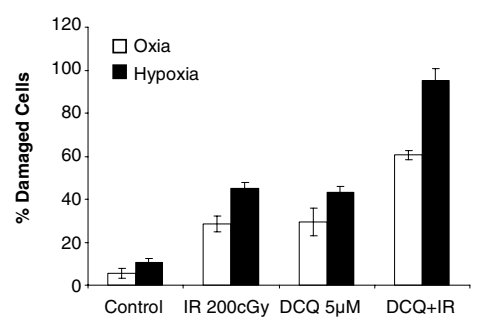

C

\begin{tabular}{|c|c|c|c|c|c|}
\hline & & Comet Length $(\mu \mathrm{m})$ & Tail Length $(\mu \mathrm{m})$ & \%DNA in Tail & Tail moment \\
\hline Oxia & $\begin{array}{l}\text { Control } \\
\text { IR } 200 \text { cGy } \\
\text { DCQ } 5 \mu \mathrm{M} \\
\text { DCQ + IR }\end{array}$ & $\begin{array}{c}40.95 \pm 6.72 \\
50.96 \pm 4.51 \\
46.40 \pm 9.56 \\
93.06 \pm 1.40\end{array}$ & $\begin{array}{c}3.08 \pm 2.90 \\
20.39 \pm 2.95 \\
16.96 \pm 2.48 \\
38.09 \pm 0.38\end{array}$ & $\begin{array}{c}5.95 \pm 1.65 \\
26.96 \pm 1.79 \\
28.82 \pm 1.15 \\
64.83 \pm 1.50\end{array}$ & $\begin{array}{c}0.18 \pm 0.017 \\
5.50 \pm 1.67 \\
4.89 \pm 1.06 \\
24.69 \pm 1.87\end{array}$ \\
\hline Hypoxia & $\begin{array}{l}\text { Control } \\
\text { IR } 200 \text { cGy } \\
\text { DCQ } 5 \mu \mathrm{M} \\
\text { DCQ + IR }\end{array}$ & $\begin{array}{c}45.95 \pm 1.13 \\
80.90 \pm 3.43 \\
76.40 \pm 2.96 \\
124.65 \pm 8.36\end{array}$ & $\begin{array}{c}6.08 \pm 1.03 \\
39.39 \pm 1.38 \\
28.98 \pm 2.08 \\
91.82 \pm 5.28\end{array}$ & $\begin{array}{c}7.94 \pm 1.56 \\
36.52 \pm 2.71 \\
33.28 \pm 1.97 \\
78.74 \pm 3.63\end{array}$ & $\begin{array}{c}0.48 \pm 0.09 \\
14.56 \pm 1.14 \\
9.79 \pm 1.45 \\
72.30 \pm 3.16\end{array}$ \\
\hline
\end{tabular}

\section{Figure 7}

Induction of DNA damage in DLD-I cells after treatment with DCQ, IR or combinations under oxic and hypoxic conditions. Cells were treated with $5 \mu \mathrm{M}$ DCQ for I hour, 2 Gy IR or combinations. Immediately after treatment, DNA damage was assessed using alkaline single cell microgel electrophoresis (Comet) assay as mentioned in the "Materials and methods" section. A. The figure shows different grades of DNA fragmentation in DLD-I cells. Magnification: I00×. B. An average of I00 cells per slide were counted and analyzed, and the mean of damaged cells is represented as the percentage of control untreated cells. C. Quantitative measurements of various comet assay end-points as analyzed using Comet Score software.

extent according to the cell type, and $\mathrm{G}_{2} / \mathrm{M}$ arrest and apoptosis are involved in the mechanism of radiosensitization by the drug. Interestingly, normal cells were less sensitive to DCQ sensitization than cancer cells.

Using the alkaline Comet assay, DCQ was found to be a redox-activated DNA-damaging agent when combined with radiation, with selective toxicity against hypoxic cells. Recent evidence indicates that the hypoxia selective cytotoxic activity of quinoxaline 1,4-dioxides involves enzymatic reduction of the compound to a crucial oxygen-sensitive radical intermediate capable of cleaving the DNA [7]. Many QdNOs are known as "chemical nucleases" that efficiently "nick" the DNA [20]. Most prominent among these compounds is 3-amino-1,2,4benzotriazine1,4-dioxide (tirapazamine TPZ), a heterocy- clic di-N-oxide that is selectively toxic to hypoxic tumor cells. TPZ is also involved in transferring oxygen atoms from its $\mathrm{N}$-oxide functional groups to these radicals, converting them to base-labile strand cleavage sites [7].

A significant increase in DNA single strand breaks, measured as alkaline tail moment, was observed in DLD-1 cells exposed to DCQ and IR under hypoxic conditions. However, DCQ and IR under oxic conditions predominantly induced relatively non-cytotoxic single-strand breaks. DNA single strand breaks or alkali labile sites are by far the largest number of lesions in DNA in general. Therefore, the decrease in cell survival and induction of apoptosis in DLD-1 cells was likely due to the additive effects of DNA damage produced by DCQ and IR upon hypoxia. On the basis of structural correlation between TPZ and the qui- 
A

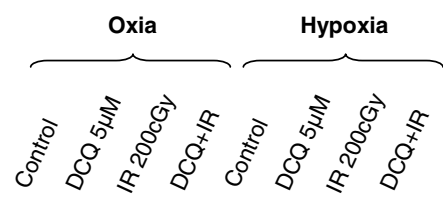

p53

p-p53

p21

GAPDH

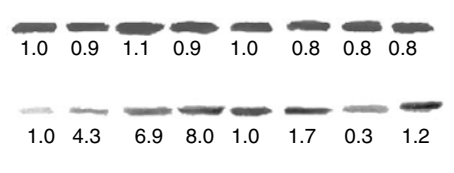

$\begin{array}{llllllllll}1.0 & 2.0 & 1.2 & 3.7 & 1.0 & 0.4 & 0.8 & 1.5\end{array}$
B

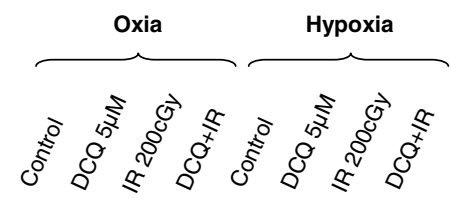

Bax

$\mathrm{Bcl}-2$

$\mathrm{Bax} / \mathrm{Bcl}-2$

GAPDH

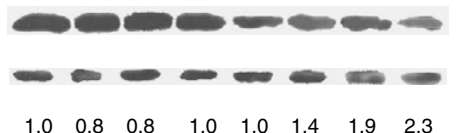

C

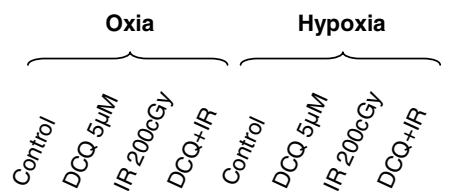

Cox-2

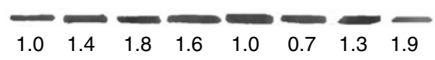

p-Akt

$\overline{1.0} \overline{1.5} \underset{1.9}{\frac{1.4}{1.0}} \overline{1.4} \frac{}{0.6} \overline{1.0}$

GAPDH

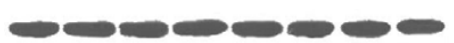

\section{Figure 8}

Effects of DCQ and IR on the expression levels of p53, p-p53, p2I (A), Bax/Bcl2 (B), p-Akt and Cox-2 (C) proteins. DLD-I cells were treated under oxic and hypoxic conditions with $5 \mu \mathrm{M} D C Q, 2$ Gy IR or combinations. After 24 hours, $40 \mu \mathrm{g}$ cell lysates were subjected to SDS-PAGE. Fold induction of protein levels was based on densitometry measurments. Protein levels in treated cells were defined as percentage of control. All plots were re-probed with GAPDH to ensure equal protein loading.

noxaline 1,4-dioxide DCQ, the latter compound can be considered as a more potent DNA radical oxidant by oxidizing such DNA radicals to cytotoxic DNA strand break [3].

Studies have reported the influence of the cellular p53 status on radiosensitivity, due to the function of this tumor suppressor gene in the cellular response to DNA damage [21]. Activation of p53 following genotoxic damage is achieved by the induction of $\mathrm{p} 53$ levels and by the phosphorylation of the p53 protein, in particular, at serine 15 and 20 [22]. Here we show that irradiating hypoxic DLD1 cells reduced the protein expression levels of p-p53, while DCQ in combination with IR caused no changes in p53 or p-p53 protein. This suggests that the enhanced response of hypoxic DLD-1 cells to the combination treatments is probably due to the radiation-induced reduction of p53 as a result of increased DNA instability at various loci [23]. However, p-p53 protein levels were increased in DLD-1 cells treated with DCQ and IR under oxic condi- tions, indicating that p53 may be involved in the mechanism by which DCQ and IR induce cell cycle arrest at $\mathrm{G}_{2} /$ $M$ phase; the most radiosensitive phase of the cell cycle.

The mechanism by which p53 induces cell-cycle arrest is highly dependent upon the transcriptional induction of p21, which inhibits cyclin dependent kinase activity that is necessary for $G_{2} / M$ transitions [24]. Our findings show that p53-p21 signaling pathways may be involved in DCQ radiosensitization under oxia but not under hypoxia in DLD-1 cells. This indicates that hypoxia enhances DCQ's potent activity as radiosensitizer through a different mechanistic pathway than what is observed under oxia.

It appears that the induction of cell death in hypoxic DLD1 cells after combination treatments involves the induction of $\mathrm{Bax} / \mathrm{Bcl}-2$ expression levels. Among the variety of proteins that control the apoptotic program are the members of the Bcl-2 family that act as inhibitors (Bcl-2, Bcl-Xl and $\mathrm{Bcl}-\mathrm{W})$, and those that act as promoters of apoptosis 
(Bax, Bad, Bak and Bcl-Xs) [25]. We showed that hypoxia enhances the expression of $\mathrm{Bcl}-2$ protein and reduces $\mathrm{Bax}$ protein expression levels, thereby inhibiting apoptosis. DNA damage could trigger apoptosis via a p53-mediated pathway that includes the upregulation of the pro-apoptotic protein Bax [26]. In our study, treatment with DCQ plus radiation under hypoxic conditions in DLD-1 cells down regulated the protein expression levels of Bax. This is further confirmation that p53 may not be involved in the induction of apoptosis by DCQ in hypoxic DLD-1 cells. Alternatively, apoptosis triggering via $\mathrm{Bax} / \mathrm{Bcl}-2$ induction might arise via another pathway.

In addition, DCQ radiosensitization effects were found to be associated with changes in the Cox- 2 signaling molecule. The anti-apoptotic Cox-2 is an enzyme that converts arachidonic acid to prostaglandins, and is inducible by various stimuli including interleukin-1, hypoxia, radiation, epidermal growth factor, transforming growth factor- $\beta$, tumor necrosis factor- $\alpha$, and several oncogenes [16]. Recent evidence suggests that Cox-2 inhibition may arrest cells in $G_{2} / M$ phase through p53 inactivation. However, in the present study, Cox-2 does not appear to be involved in $G_{2} / M$ phase arrest of DLD-1 cells when combination treatments were done under oxia, as p-p53 protein expression levels were induced. Since the modulation of protein expression levels was studied in DLD-1 cells, these results may not be extrapolated to other colon cancer cell lines that showed different features with regard to hypoxic radiosensitization.

Our present data show that pretreatment with DCQ under hypoxic conditions induces cell death in DLD-1 cells probably through the reduction of Cox-2 protein. One mechanism for the pro-apoptotic activity of Cox-2 has been the down-regulation of $\mathrm{Bcl}-2$. Although the precise link between Cox-2 and Bcl-2 has not been elucidated, it is interesting to speculate on the potential role of DCQ in enhancing the sensitivity of hypoxic DLD-1 cells to radiation upon the inhibition of Cox-2 and $\mathrm{Bcl}-2$. More recently, celecoxib, a potent and selective Cox- 2 inhibitor, was shown to induce apoptosis in human prostate cancer cells by blocking Akt activation, independent of $\mathrm{Bcl}-2$ signaling [27]. Our results correlate with that of celecoxib, since hypoxic treatment with DCQ inhibited the phosphorylation of the Akt prosurvival gene upon IR exposure. Evidence suggests that the Akt/PKB pathway promotes growth factor-mediated cell survival and inhibits apoptosis via modifying the anti-apoptotic and pro-apoptotic activities of members of the $\mathrm{Bcl}-2$ gene family [16]. Cox-2 may represent a downstream mediator of the Akt/PKB pathways.

\section{Conclusion}

In summary, the data presented here indicate that DCQ could be used as a model radiosensitizer to understand the crosstalk between signaling molecules involved in radiation enhancement. This hypoxic cell radiosensitizer is a potentially useful drug that enhances the response of DLD-1 human colon cancer cells to IR. The radiosensitizing efficacy of DCQ is related to the oxygenation status of the cell and the type of tumor cell. In addition, DCQ seems to generate lethal single stranded DNA breaks upon IR exposure. DCQ radiosensitization effects in DLD-1 cells occur mostly through the enhanced induction of $\mathrm{G}_{2} /$ $M$ arrest under oxia and apoptosis induction under hypoxia. Apoptosis by DCQ in DLD-1 cells is associated with the inhibition of Cox-2 protein levels and the increase in $\mathrm{Bax} / \mathrm{Bcl}-2$ ratio.

\section{Competing interests}

The author(s) declare that they have no competing interests.

\section{Authors' contributions}

WI participated in the design of the study, contributed to data acquisition and analysis and in drafting the paper. FG was involved in revising the manuscript critically for important intellectual content. JH participated in performing the Comet assay. MH provided the compound and critically reviewed the manuscript. HM conceived of the study, and participated in its design and coordination and drafted the manuscript. All authors read and approved the final manuscript.

\section{Acknowledgements}

This study was supported by the University Research Board of the American University of Beirut and the Lebanese National Council for Scientific Research. We thank members of the Central Research Science Laboratory for helping with flow cytometry.

\section{References}

I. Hockel M, Vaupel P: Tumor Hypoxia: Definitions and Current Clinical, Biologic, and Molecular Aspects. I Natl Cancer Inst 200I, 93:266-276.

2. Vaupel P: Tumor Microenvironmental Physiology and its Implications for Radiation Oncology. Semin Radiat Oncol 2004, 14:198-206.

3. Weinmann M, Welz S, Bamberg M: Hypoxic radiosensitizers and hypoxic cytotoxins in radiation oncology. Curr Med Chem: AntiCancer Agents 2003, 3:364-374.

4. Phillips RM, Jaffar M, Maitland DJ: Pharmacological and biological evaluation of a series of substituted I,4-naphthoquinone bioreductive drugs. Biochem Pharmacol 2004, 68:2107-2116.

5. Brown JM: Exploiting the hypoxic cancer cell: mechanisms and therapeutic strategies. Mol Med Today 2000, 6:157-162.

6. Ganley B, Chowdhury G, Bhansali J, Daniels JS, Gates KS: RedoxActivated, Hypoxia-Selective DNA Cleavage by Quinoxaline I,4-di-N-Oxide. Bioorganic \& Medicinal Chemistry 200I, 9:2395-240I.

7. Ortega MA, Morancho MJ, Martinez-Crespo FJ: New quinoxaline carbonitrite I,4-di-N-oxide derivatives as hypoic cytotoxic agents. Eur J Med Chem 2000, 35:2I-30. 
8. Diab-Assef M, Haddadin M, Yared P, Assaad C, Gali-Muhtasib H: Quinoxaline I,4-Dioxides: Hypoxia-Selective Therapeutic Agents. Molecular Carcinogenesis 2002, 33:198-205.

9. Gali-Muhtasib H, Sidani M, Geara F, Assaf-Diab M, Al-Hmaira J, Haddadin M, Zaatari G: Quinoxaline I,4-dioxides are novel angiogenesis inhibitors that potentiate antitumor effects of ionizing radiation. Int J Onco 2004, 24: I | | I- I I3I.

10. Haddadin M, Issidorides C: The Beirut Reaction. Heterocycles 1993, 35:1503-1525.

II. Dhawan A, Bajpayee M, Pandy AK, Parmar D: Protocol For The Single Gel Electrophoreisis/Comet Assay For Rapid Genotoxicity Assessment. ITRC: The SCGE/Comet Protocol 2005.

12. Kubbutat M, Jones S, Vousden K: Regulation of p53 stability by Mdm2. Nature 1997, 387:299-303.

13. El-Deiry W: Regulation of p53 downstream genes. Cancer Biol 1998, 8:345-357.

14. Brugarolas J, Moberg K, Boyd S: Inhibition of cyclin-dependent kinase 2 by $\mathrm{p} 2 \mathrm{I}$ is necessary for retinoblastoma proteinmediated GI arrest after gamma-irradiation. Proc Natl Acad Sci USA 1999, 961:1002-1007.

15. Fukuchi K, Watanabe H, Tomoyasu S: Phosphotidyl 3-kinase inhibitors, Wortmannin or LY294002 inhibited accumulation of p2I protein after $\gamma$-irradiation by stabilization of the protein. Biochim Biophys Acta I496:207-220.

16. Davis TW, Hunter N, Trifan OC, Milas L, Masferrer JL: COX-2 Inhibitors as Radiosensitizing Agents for Cancer Therapy. Am J Clin Oncol (CCT) 2003, 26:S58-S6I.

17. Ueta $\mathrm{E}$, Yoneda $\mathrm{K}$, Kimura : Mn-SOD antisense upregulates in vivo apoptosis of squamous cell carcinoma cells by anticancer drugs and $\gamma$-rays regulating expression of the BcL-2 family proteins, COX-2 and p2I. Int J Cancer 200I, 94:545-550.

18. Liu X, Kirschenbaum A, Yu K, Yao S, Levine A: Cyclooxygenase-2 Suppresses Hypoxia-induced Apoptosis via a Combination of Direct and Indirect Inhibition of p53 Activity in a Human Prostate Cancer Cell Line. J Biol Chem 2005, 280:38I7-3823.

19. Kaanders J, Bussink J, van der Kogel A: Clinical Studies of Hypoxia Modification in Radiotherapy. Semin Radiat Oncol 2004, 14:233-240.

20. Staszewska A, Stefanowicz P, Szewczuk Z: Direct solid-phase synthesis of quinoxaline-containing peptides. Tetrahedron Lett 2005, 46:5525-5528.

21. Matsui Y, Tsuchida Y, Keng PC: Effects of p53 mutations on cellular sensitivity to ionizing radiation. Am J Clin Oncol 200I, 24:486-90.

22. Chandel NS, Vander HM, Thompson CB, Schumacker PT: Redox regulation of $\mathrm{p} 53$ during hypoxia. Oncogene 2000, 19:3840-8.

23. Samuni A, Kasid U, Chuang E: Effects of Hypoxia on RadiationResponsive Stress-Activated Protein Kinase, p53, and Caspase 3 Signals in TK6 Human Lymphoblastoid Cells. Cancer Res 2005, 65:579-86.

24. El-Deiry W: The role of $\mathbf{p 5 3}$ in chemosensitivity and radiosensitivity. Oncogene 2003, 22:7486-7495.

25. Cuisnier O, Serduc R, Lavieille JP, Longuet M, Reyt E, Riva C: Chronic hypoxia protects against $\gamma$-irradiation-induced apoptosis by inducing bcl-2 up-regulation and inhibiting mitochondrial translocation and conformational change of bax protein. Int J Onco 2003, 23: 1033-104I.

26. Prise KM, Schettino G, Folkard M, Held KD: New insights on cell death from radiation exposure. Lancet Oncol 2005, 6:520-28.

27. Choy H, Milas L: Enhancing Radiotherapy With Cyclooxygenase-2 Enzyme Inhibitors: A Rational Advance? J Natl Cancer Inst 2003, 95: 1440-52

\section{Publish with Biomed Central and every} scientist can read your work free of charge

"BioMed Central will be the most significant development for disseminating the results of biomedical research in our lifetime. "

Sir Paul Nurse, Cancer Research UK

Your research papers will be:

- available free of charge to the entire biomedical community

- peer reviewed and published immediately upon acceptance

- cited in PubMed and archived on PubMed Central

- yours - you keep the copyright
BioMedcentral 